\title{
Reducing Driving Violations by Receiving Feedback from Other Drivers
}

\author{
Chao Wang \\ Eindhoven University of \\ Technology \\ Eindhoven, the Netherlands \\ ch.wang@tue.nl

\section{Jacques Terken} \\ Eindhoven University of \\ Technology \\ Eindhoven, the Netherlands \\ j.m.b.terken@tue.nl

\section{Bin Yu} \\ Eindhoven University of \\ Technology \\ Eindhoven, the Netherlands \\ B.Yu@tue.nl
}

Permission to make digital or hard copies of part or all of this work for personal or classroom use is granted without fee provided that copies are not made or distributed for profit or commercial advantage and that copies bear this notice and the full citation on the first page. Copyrights for third-party components of this work must be honored. For all other uses, contact the Owner/Author.

Copyright is held by the owner/author(s).

AutomotiveUI '15 Adjunct, September 01-03, 2015,

Nottingham, United Kingdom

ACM $978-1-4503-3858-5 / 15 / 09$

http://dx.doi.org/10.1145/2809730.2809736

\begin{abstract}
The road environment can be seen as a social situation and road user safety can be viewed as not just skillsbased and rule-governed. Numerous studies show that intentional driving violations make an independent significant contribution to traffic accident involvement. In this study, we propose a concept based on Driver to Driver communication and social network, which enables road users to express their anger and appreciation to others' driving behavior. Investigation on how drivers feel about receiving feedback of evaluating their driving behavior from other drivers was conducted. The results show that such evaluation has positive influence on reducing driving violations.
\end{abstract}

\section{Author Keywords}

Connected car; driving violations; $\mathrm{C} 2 \mathrm{C}$ communication; social pressure.

\section{ACM Classification Keywords}

H.4.m [Information Systems Applications]:

Miscellaneous; H.5.2 [Information Systems

Applications]: User Interfaces - user-centered design

\section{Introduction}

Like using other communal facilities, drivers need to coordinate with each other to share the road infrastructure [1]. But when we sit in our "iron cages", there are only a few channels to deliver information on the road. Cars appear as machines, lacking any 


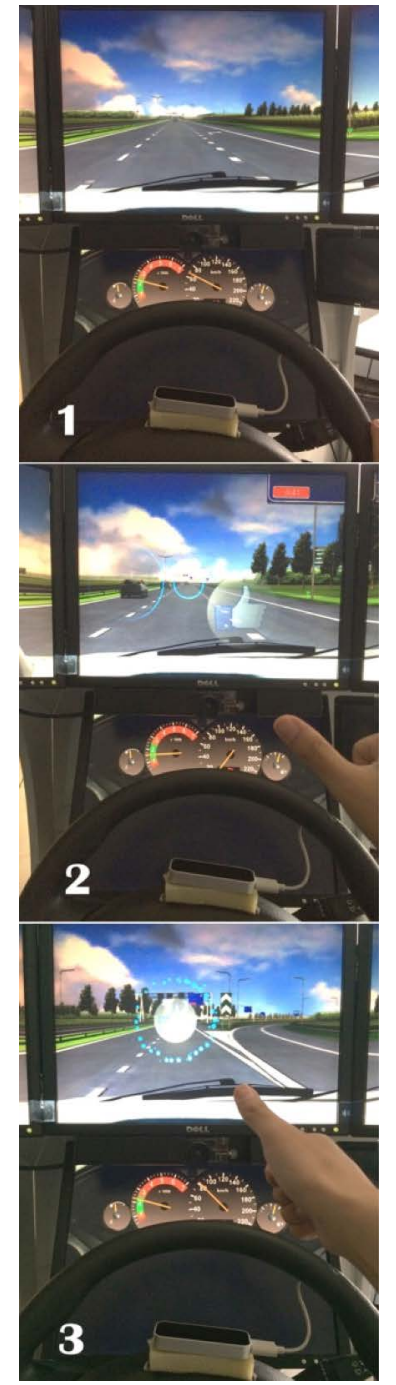

Figure 1. Sending "Like" and "Dislike" to others by gestures anthropomorphic (human-like) features [2]. This lack has also been shown to contribute towards aggressive, selfish and anti-social driving behavior [3]. Recently, this situation has been changed by everywhere available connectivity of internet and the broad penetration of social network services [4]. The relationship between drivers on the road may gain more transparency, enabling social information to pass through the steel shell of the cars and giving opportunities to reduce anonymity and strengthen empathy.

Previously, we proposed a system based on Driver to Driver communication and social network, which enabled users to express appreciation of nearby driver's polite behavior on the road [5]. A gesture based interaction system for this concept was evaluated through a user test deploying a prototype integrated in a driving simulator (Fig. 1). The results demonstrated that people held a positive attitude towards this concept as well as the gestural interaction design.

In the present study, we changed the perspective and investigated how drivers felt about receiving feedback from other drivers to evaluate their driving behavior, for the purpose of examining whether such evaluation would have a positive influence on reducing driving violations.

\section{Related work}

Driving violations and social influences

The road environment can be seen as a social situation, with actors or agents that interact and influence one another [2]. For understanding of road user safety and interventions, the social nature of driving activity should be taken into account [6]
Different models have been used to explain the social influences on driving behavior. The Theory of Planned Behavior (TPB) has a long tradition of being used in explaining individual factors that influence road user safety behavior [7][8][9]. Parker et al. argued that the relationship between subjective norms and behavioral intentions to commit driving violations was consistently stronger than between attitudes towards behavior and behavioral intentions [10]. In their study with 881 drivers they showed that the social aspect had a strong influence on road user safety behavior [11]. Recently, Bronfenbrenner's Ecological System Theory model [12]. Musselwhite et al. investigated 228 drivers by applying this model in the analysis of participants' speeding behavior [6]. They argued that peer pressure has a strong influence on driving behavior, even if others are not physically present. "Soft" behavioral change interventions, from different layer of social influences based on this model were suggested.

Concept and hypothesis

In this study, we investigated the effectiveness of a social intervention to reduce drivers' violations on the road. In a previous study the idea of "Liking other drivers' behavior" emerged as a promising concept from discussions with more than twenty people. The concept held that "You can give other drivers a "Like" or a "Dislike". With respect to sending "Like" or "Dislike" on the road, the results of an evaluation of a prototype based on gesture interaction demonstrated that drivers were able to give specific driver's real time feedback of their driving behavior. But whether feedback from other road users will exert positive influence on people's driving behavior is still unknown. Further more, punishments communicate obligatory rules while rewards communicate voluntary rules [13]. 


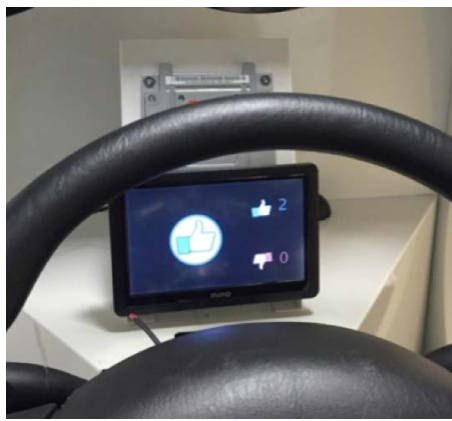

Figure 2: An 8" screen was set in front of the steering wheel to show other driver's evaluation.

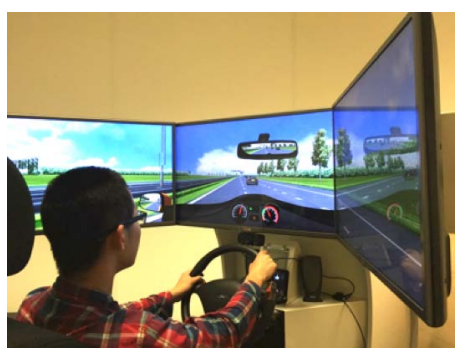

Figure 3: Participants were asked to complete highway driving task for 3 times: with both positive and negative feedback, with only positive feedback and without any feedback.
As a result, we tested 2 hypotheses:

- H1: The presence of real time feedback has positive influence on driving behavior. It was predicted that the participants' driving behavior improved according to both objective and subjective evaluation of driving performance.

- H2: Dual feedback (positive + negative) and only positive feedback exert different influence on driver's behavior. It was predicted that driving behavior data are different.

\section{Methodology}

Apparatus

A setup integrating an 8 " screen and an advanced driving simulator was adopted to evaluate this concept (Fig. 2). The driving simulator system included a steering wheel, seat, pedals, gears and three 42" screens. When the participants were driving in the simulator, the driving image was also displayed on the screen in another room, enabling an "observer" to observe the driving behavior and giving evaluative feedback about participants' driving behavior. If the observer clicked the "Like" or "Dislike" icon on the screen of a laptop that was connected to the 8 " screen in front of the participant, the corresponding icon appeared to inform him though visual and sound feedback.

\section{Experiment Design}

A within group test, which involved 18 participants (16 males, 2 females; ranging from 21 to 32 with mean 24.5, all with more than 3 years driving experience), was conducted in this study. A highway scenario, which included curves, viaducts, entrance ramps and exit ramps, along with high density of traffic setting was created for testing. A navigator system was also integrated.

An observer, who acted as "other road drivers" by supervising their behavior from a monitor, evaluated "Like" and "Dislike" evaluation in a separate room. The standard of negative and positive evaluation was created based on "20 most annoying driving behaviors" in the survey conducted by the Consumer Reports National Research Center in January 2012 [14]. The observer followed the criterion as below:

For evaluating behavior as "dislike"

- Tailgating

- Cutting off other cars

- Speeding and swerving in and out of traffic

- Not letting others merge into a lane

- Not using turn signals

- Driving slowly dawdling in the passing lane

- Indecisive about where to turn

For evaluating behavior as "Like":

- Giving space to others by changing to overtaking lane when they are merging into highway

- Reducing speed gently

- Letting fast car overtaking

- Keeping distance to front vehicle when they reduce speed

At first, each participant was invited to drive on the simulator in a free driving mode for 30 minutes with the purpose of getting familiar with the driving simulator. Then she/he was introduced to the concept 

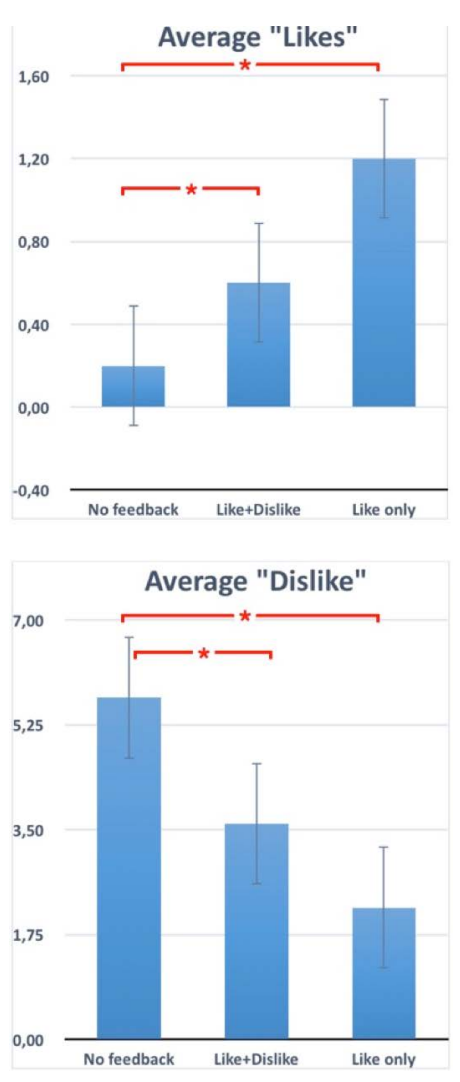

Figure 4: Average "Likes" and "Dislike" allocated in three conditions. * indicates significant difference. of the driving behavior credit system. Then the participants were asked to assume themselves driving under this condition: You are driving to meet a client in an unfamiliar city. It's a little late. Unfortunately, you encounter heavy traffic on the highway, so follow the navigator and drive as fast as you can.

All the participants were asked to complete three driving tasks, each lasting 15 minutes (Fig. 3). The observer observed the behavior and evaluated it as "Like" and "Dislike" when they driving. Actually, the observer gave and recorded "Like" and "Dislike" in all three conditions, even though participants conditionally received this feedback in 3 driving tasks: In the "Like + Dislike" condition, they received "Like" and "Dislike" feedback in real time; In the "Like only" condition, they received only "Like" and in the "No feedback" condition, they received no feedback. Some kinds of negative emotional expression, for example obscene gesture and beeps, which can be regarded as "directly hostility" may lead to aggressive behavior per se [15]. For this reason, the experiment condition with only "Dislike" feedback was not included in this study. The sequence of the tasks was randomized to balance for carry-over effects. Furthermore, 18 participants were distributed equally to 6 groups for counterbalancing. An interview were followed when they finishing all the tasks.

\section{Results}

RM-ANOVA was conducted to compare their social driving behavior, which according to subjective evaluation from the observer and objective driving performance generated by driving simulator, in three conditions: (i) no feedback; (ii) "Like" and "Dislike" feedback and; (iii) only "Like" feedback.
Observer of driving performance

There was significant difference of "Like", $F(2,18)=$ $5.104, p=0.018$ and "Dislike", $F(2,18)=4.791, p=$ 0.021 , evaluation that participants received in three conditions. For "Like", there was significant difference between the group of no feedback and the group of only receiving positive feedback ( $p=0.023)$, but there is no significant difference between "No feedback" and "Like + Dislike" groups $(p=0.168)$, neither between "Like + Dislike" and "Like only" ( $p=0.81)$. For "Dislike", there was significant difference between "Like only" and "No feedback" groups ( $p=0.019)$, as well as between "Like + Dislike" and "No feedback" ( $p=$ $0.032)$. However, there is no differences between "Like + Dislike" and "Like only" ( $p=0.275)$. Fig.4 shows the mean of driver received "Dislike" and "Like" in three conditions. This indicated that drivers behaved more politely when they got feedback.

Objective driving performance

GreenDino's driving simulator system was able to generate 30 kinds of scores by analyzing the data of user's performance. Five of them (Keeping safe speed, Keeping fluent speed, Position inside lane, Smooth steering and Keeping distance to preceding car), which related to social driving behavior were selected as criteria for objective evaluation. Each score ranges from 0 to 10 and a higher value indicates better performance. The social driving performance was the average of five items. Fig. 5 shows the mean of all the scores, the mean of driving performance with feedback is higher than no feedback. However, there is no significant difference of there objective driving performance in three conditions: $F(2,18)=3.463, p=$ 0.053 . 


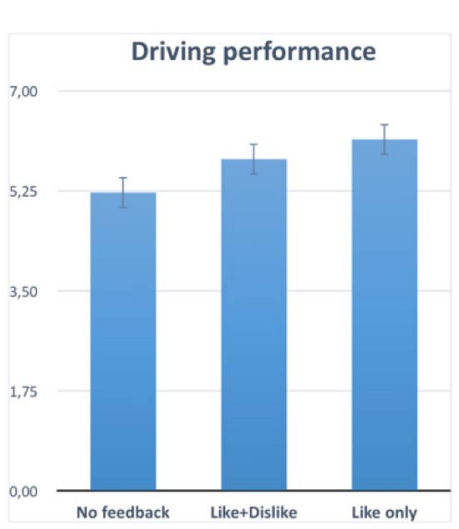

Figure 5: Average driving performance data generated by simulator in three conditions.

\section{In-depth interview}

In order to also get further feedback about this system, in-depth interviews were conducted after the experiment. Participants were asked several questions about their opinion of the influence on driving and whether they would be willing to use such system.

All participants were willing to express their anger and appreciation to other road users and receive others' evaluation as well. Many participants suggested that a mechanism should be established to prevent abuse of "Dislike". Further punishment (e.g. related to insurance fee) and rewarding policies were accepted, but comparing to material benefit, a few of them preferred more "soft" solution such as a virtual badge on the social network.

When asked about whether they would improve their driving behavior when they got "Dislike", most of the participants said they were more careful after receiving negative feedback and this did not infuriate them. "I am not angry about other's evaluation. They remind me to take care of my safety" (participant 8).

One of the participants thought that showing other driver's behavior would exert positive influences on him. "Maybe I will be better if I know I got much more bad feedback than average, especially after showing me some demonstration of the correlation between this and accident rate" (participant 18).

Most participants reported that they were not able to realize the reason of getting each "Like" and "Dislike". "I need more information to know what happened. For example, video record and driving data" (participant 10). Furthermore, three participants thought that receiving evaluation in real time, especially negative feedback, distracted their driving. They preferred to obtain feedback afterwards.

As regards the different impacts between "Like" and "Dislike" evaluation, about half of all participants said they drove more carefully in order to get the "rewarding" from others. "I have tried very hard to get a "Like". It makes me happy!" (participant 7).

\section{Conclusion and future work}

In this study, we investigate how drivers feel about receiving feedback evaluating their driving behavior from other drivers. We found support for the following hypotheses

- H1. The results gave partly support for $\mathrm{H} 1$. Participants drove more social according to subjective evaluation, but there is no significant difference of objective driving performance in three conditions. Indepth interview provided more evidence that real time feedback improved drivers' social awareness, even though there was no material benefit.

- H2. According to the result of the evaluation, there is no differences between positive feedback and dual feedback.

In previous work, sending feedback to other drivers by gesture was shown to be appreciated. In this study, the improvement of driving behavior after receiving feedback was not rejected. In the future, according to the requirement of participants, an afterwards feedback, which enables drivers to reflect on their driving behavior by reviewing videos and driving data that show why they received "Like" and "Dislike" will be implemented (Fig. 6). Furthermore, other social communication channels such as leaving comments afterwards will be implemented in order to exert more 
influences on drivers' behavior.
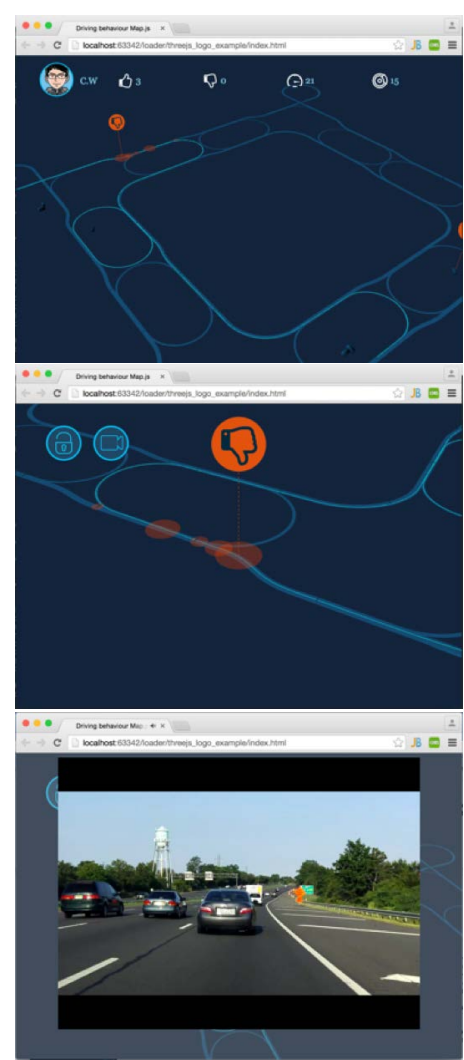

Figure 6: An interactive website which enable participants to review their driving data and video record to reflect why they got "Like" and "Dislike".

\section{References}

1. Juhlin, O. (2010). Social media on the road: the future of car based computing (Vol. 50). Springer Science \& Business Media.

2. Haglund, Mats, and Lars Åberg. "Speed choice in relation to speed limit and influences from other drivers." Transportation Research Part F: Traffic Psychology and Behaviour 3.1 (2000): 39-51.

3. Rakotonirainy, A., Feller, F., \& Haworth, N. L. (2008). Using in-vehicle avatars to prevent road violence.

4. Schroeter, R., Rakotonirainy, A., \& Foth, M. (2012, October). The social car: new interactive vehicular applications derived from social media and urban informatics. In Proceedings of the 4th International Conference on Automotive User Interfaces and Interactive Vehicular Applications (pp. 107-110). ACM.

5. Wang, C., Terken, J., \& Hu, J. (2014, September). Liking other Drivers' Behavior while Driving. In Adjunct Proceedings of the 6th International Conference on Automotive User Interfaces and Interactive Vehicular Applications (pp. 1-6). ACM.

6. Musselwhite, C., Avineri, E., \& Susilo, Y. O. (2014). Legitimising risk taking: articulating dangerous behaviour on the road. Transportation planning and technology, 37(1), 62-82.

7. Armitage, C. J., \& Conner, M. (2001). Efficacy of the theory of planned behaviour: A meta-analytic review. British journal of social psychology, 40(4), 471-499.

8. Conner, M., Lawton, R., Parker, D., Chorlton, K., Manstead, A. S., \& Stradling, S. (2007). Application of the theory of planned behaviour to the prediction of objectively assessed breaking of posted speed limits. British Journal of Psychology, 98(3), 429453.

9. Elliott, M. A., Armitage, C. J., \& Baughan, C. J. (2003). Drivers' compliance with speed limits: an application of the theory of planned behavior. Journal of Applied Psychology, 88(5), 964.

10. Manstead, A. S., Parker, D., Stradling, S. G., Reason, J. T., \& Baxter, J. S. (1992). Perceived consensus in estimates of the prevalence of driving errors and violations. Journal of Applied Social Psychology, 22(7), 509-530.

11. Parker, D., Manstead, A. S., Stradling, S. G., \& Reason, J. T. (1992). Determinants of intention to commit driving violations. Accident Analysis \& Prevention, 24(2), 117-131.

12. Bronfenbrenner, U., \& Morris, P. A. (1998). The ecology of developmental processes.

13. Mulder, L. B. (2008). The difference between punishments and rewards in fostering moral concerns in social decision making. Journal of Experimental Social Psychology, 44(6), 1436-1443.

14. Survey reveals top gripes among drivers. Retrieved March, 2012 from

http://www.consumerreports.org/cro/2012/03/surv ey-reveals-top-gripes-among-drivers/index.htm

15. Lajunen, T., \& Parker, D. (2001). Are aggressive people aggressive drivers? A study of the relationship between self-reported general aggressiveness, driver anger and aggressive driving. Accident Analysis \& Prevention, 33(2), 243-255. 
Adjunct Proceedings of the $7^{\text {th }}$ International Conference on Automotive User Interfaces and Interactive Vehicular Applications (AutomotiveUI '15), Sept. 1-3, 2015, Nottingham, United Kingdom

The Association for Computing Machinery

2 Penn Plaza, Suite 701

New York New York 10121-0701

ACM COPYRIGHT NOTICE. Copyright @ 2015 by the Association for Computing Machinery, Inc.

Permission to make digital or hard copies of part or all of this work for personal or classroom use is granted without fee provided that copies are not made or distributed

for profit or commercial advantage and that copies bear this notice and the full citation on the first page. Copyrights for components of this work owned by others than ACM must be honored. Abstracting with credit is permitted. To copy otherwise, to republish, to post on servers, or to redistribute to lists, requires prior specific permission and/or a fee. Request permissions from Publications Dept., ACM, Inc., fax +1(212)869-0481, or permissions@acm.org.

For other copying of articles that carry a code at the bottom of the first or last page, copying is permitted provided that the per-copy fee indicated in the code is paid through the Copyright Clearance Center, 222 Rosewood Drive, Danvers, MA 01923, +1-978-750-8400, +1-978-750-4770 (fax).

\section{Notice to Past Authors of ACM-Published Articles}

ACM intends to create a complete electronic archive of all articles and/or other material previously published by ACM. If you have written a work that was previously published by ACM in any journal or conference proceedings prior to 1978 , or any SIG Newsletter at any time, and you do NOT want this work to appear in the ACM Digital Library, please inform permissions@acm.org, stating the title of the work, the author(s), and where and when published.

ACM ISBN: 978-1-4503-3736-6 


\section{Preface}

We are very pleased to introduce the Adjunct Proceedings of the Seventh International Conference on Automotive User Interfaces and Interactive Vehicular Applications (AutomotiveUI'15). This is the premier forum for user interface (UI) research in the automotive domain. As with previous conferences, the papers and presentations as part of AutomotiveUI'15 addresses novel in-vehicle services, models of and concepts for enhancing the driver experience, driver performance and behavior, development of semi and fully autonomous driving, and the needs of different user groups.

It is widely recognized that the automobile is progressing towards the status of "computer on wheels" with greater connectivity to the outside world and higher levels of autonomy. As a consequence, not only will what we consider to be "driving" fundamentally change, but also a plethora of novel functions and services will become available to the users of future vehicles. The design of the automotive user-interface is/will be complex, dependent on many 'hard' (e.g. performance, safety) and 'soft' (e.g. likes/ dislikes) variables. The papers within this year's Automotive $\mathrm{UI}$ conference reflect this breadth and depth of issues, ranging from specific usercentred issues facing industry now (e.g. relating to evaluation of distraction), through to longerterm perspectives, such as how to design Uls for car interiors in which the "driver" spends large amounts of time not in control of his/her vehicle.

Automotive UI'15 is hosted by the Human Factors Research Group (HFRG), Faculty of Engineering at the University of Nottingham. The group conducts research into the human factors (ergonomics) issues for new technology, across a range of domains including transport (road and rail), healthcare, manufacturing, education, etc. Specific to this conference, HFRG investigates the user-interface design and evaluation issues for future vehicles, often using simulation as a safe, controlled and cost-effective environment.

\section{Submission and Review Process}

Authors were invited to submit position papers for work in progress (WIP) and demonstrations in the Extended Abstract Format. There were 38 WIP and 4 demonstrations submitted. All submissions received at least three independent, expert reviews. Based on the reviews, the WIP/Demonstration Chairs selected 26 WIP (68\%) and two contributions in the interactive demonstration category (50\%) for inclusion in the adjunct proceedings. The conference was organized in cooperation with the Association for Computing Machinery (ACM), so that the proceedings and adjunct proceedings will be available through the ACM digital library portal. 


\section{Acknowledgements}

I would like to recognize the co-chairs for the four tracks of the conference: Papers, Work-inProgress \& Demonstrations, Workshops, and Doctoral Colloquium. These experts in our field committed a considerable amount of time to promote the conference, to organize the submission and review process, and were instrumental in making decisions for the final conference program. I would like to thank the Publication Co-Chairs, the Local Arrangements Chair, as well as the webmasters for their contributions in organizing the conference.

I also thank all the members of the Program Committee for their time and effort in evaluating the many paper submissions, work-in-process papers, and demonstrations. I would like to express my sincere gratitude for their efforts in carefully evaluating the novelty and contribution of each paper, and for providing thoughtful comments to support their evaluations.

Finally, I would like to thank the University of Nottingham staff and student volunteers for their help with the local organization of the conference.

Thanks again for everything. I could not have done it without all of you.

General Chair

Gary Burnett, University of Nottingham 
Adjunct Proceedings of the $7^{\text {th }}$ International Conference on Automotive User Interfaces and Interactive Vehicular Applications (AutomotiveUI '15), Sept. 1-3, 2015, Nottingham, United Kingdom

\section{Conference Organization}

\section{General Chair}

Gary Burnett, University of Nottingham, UK

\section{Program Co-Chairs}

Joe Gabbard, Virginia Tech, US

Paul Green, University of Michigan Transportation Research Institute (UMTRI), US

Sebastian Osswald, Technische Universitat Munchen (TUM), Germany

\section{Workshop Co-Chairs}

Alexander Meschtscherjakov, University Salzburg, Austria

Peter Frohlich, Telecommuncations Research Center Vienna (FTW), Austria

\section{Doctoral Colloquium Co-Chair}

Andreas Riener, Johannes Kepler University Linz, Austria

\section{Work in Progress \& Interactive Demo Co-Chair}

Bastian Pfleging, University of Munich (LMU), Germany

Andrew L. Kun, University of New Hampshire, US

\section{Publication Co-Chairs}

Ayse Eren, University of Nottingham, UK

Vicki Antrobus, University of Nottingham, UK

\section{Webmasters}

Andreas Löcken, University of Oldenburg, Germany

Bastian Pfleging, University of Munich (LMU), Germany

\section{Local Arrangement Co-Chair}

Lesley Gray, University of Nottingham, UK

Diane Karim, University of Nottingham, UK 


\section{Adjunct Proceedings of the $7^{\text {th }}$ International Conference on Automotive User Interfaces and Interactive Vehicular Applications (AutomotiveUI '15), Sept. 1-3, 2015, Nottingham, United Kingdom \\ Program Committee}

Ignacio Alvarez, Intel Corporation

Timo Baumann, Universität Hamburg

Axel Baumgartner, University of Salzburg

Patrick Bonhoure, valeo

Andreas Braun, Fraunhofer IGD

Nora Broy, BMW Research and Technology

Peter Burns, Transport Canada

Chun-Cheng Chang, University of Washington

Mario Chiesa, Istituto Superiore Mario Boella

Stefan Diewald, Technische Universität München

Ayse Eren, University of Nottingham

Jim Foley, Toyota Technical Center

Peter Fröhlich, Austrian Institute of Technology (AIT)

Thomas Gable, Georgia Institute of Technology

Magdalena Gärtner, University of Salzburg

Jan Gugenheimer, Ulm University

Wilko Heuten, OFFIS

Hyungil Kim, Virginia Polytechnic Institute and State University

Moritz Körber, Technische Universität München

Johannes Kraus, University of UIm

Sven Krome, RMIT University / GEElab Europe

Andreas Löcken, University of Oldenburg

Nikolas Martelaro, Stanford University

Alexander Meschtscherjakov, University of Salzburg

David Miller, Stanford University

Erika Miller, University of Washington

Evangelos Niforatos, University of Lugano (USI)

Ioannis Politis, University of Glasgow

Andry Rakotonirainy, QUT

Benjamin Reaves, Oracle

Diana Reich, Research Training Group Prometei (TU
Berlin)

Bryan Reimer, MIT

Florian Schaub, Carnegie Mellon University

Stefan Schneegass, University of Stuttgart

Ronald Schroeter, QUT - CARRS-Q

Fabius Steinberger, Urban Informatics Research Lab,

Queensland University of Technology

Phillip Taylor, The University of Warwick

Jacques Terken, Technische Universiteit Eindhoven

Sarah-Kristin Thiel, Ludwig-Maximilians Universität

München

Nadine Walter, Robert Bosch GmbH

Christian Wolff, Regensburg University

Yuqing Wu, University of Washington

Daniel Buschek, Ludwig-Maximilians Universität

München

Jonas Eckhardt, Technische Universität München

Abdallah El Ali, University of Oldenburg

Renate Haeuslschmid, Ludwig-Maximilians Universität

München / IAV automotive engineering

Mariam Hassib, University of Stuttgart / Ludwig

Maximilians Universität München

Sebastian Hergeth, BMW Research and Technology

Philipp Hock, University of UIm

Axel Hösl, Ludwig-Maximilians Universität München

Mohamed Khamis, Ludwig-Maximilians Universität

München

Patrick Lindemann, University of Passau

Lars Lischke, University of Stuttgart

Marcel Walch, University of UIm 
Adjunct Proceedings of the $7^{\text {th }}$ International Conference on Automotive User Interfaces and Interactive Vehicular Applications (AutomotiveUI '15), Sept. 1-3, 2015, Nottingham, United Kingdom

\section{Table of Contents}

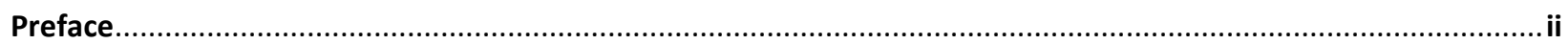

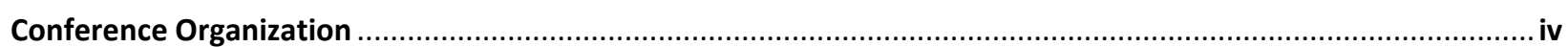

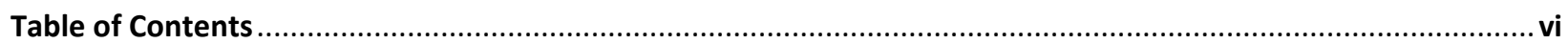

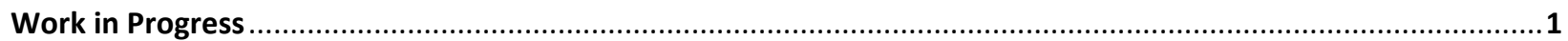

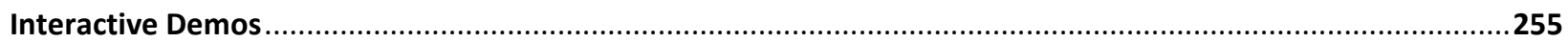

\section{Work in Progress}

Intelligent In-Vehicle Touchscreen Aware of the User Intent for Reducing Distractions:

A Pilot Study

Bashar Ahmad, Patrick Langdon, Simon Godsill, Robert Hardy and Lee Skrypchuk

Anthropomorphic Agents, Transparent Automation and Driver Personality: Towards an Integrative Multi-level Model of Determinants for Effective Driver-Vehicle Cooperation in Highly Automated Vehicles .......................... 8 Johannes Maria Kraus, Julian Elias Reiser, Jessica Sturn and Martin Baumann

User Interface Considerations to Prevent Self-Driving Carsickness

Cyriel Diels and Jelte E. Bos

Comparing Heart Rate and Pupil Size as Objective Measures of Workload in the Driving Context: Initial Look

Thomas Gable, Andrew Kun, Bruce Walker and Riley Winton

Stick'n Conversation: Stick In-car Conversation into Places using Multi Persons' Finger Pointing

Gestures

Kohei Matsumura, Tadashi Sakamoto, Haruo Noma and Yasuyuki Sumi

TactiCar: Towards Supporting Drivers During Lane Change Using Vibro-Tactile Patterns

Andreas Löcken, Hendrik Buhl, Wilko Heuten and Susanne Boll

Estimation of Drivers' Emotional States Based on Neuroergonmic Equipment: An Exploratory Study Using fNIRS

Maryam Fakhrhosseini, Myounghoon Jeon and Rahul Bose

Ghost Driver: A Platform for Investigating Interactions Between Pedestrians and Driverless Vehicles

Dirk Rothenbuecher, Jamy Li, David Sirkin, Brian Mok and Wendy Ju

"Don't Make Me Turn This Seat Around!" Driver and Passenger Activities and Positions in Autonomous Cars

Hillary Ive, David Sirkin, David Miller, Jamy Li and Wendy Ju

Advanced Traffic Light Interface: Countdown Timers to Increase User Experience

Andreas Frank, Fabian Schneider, Alexander Meschtscherjakov and Julian Stadon

Reducing Driving Violations by Receiving Feedback from Other Drivers

Chao Wang, Jacques Terken, Bin Yu and Jun Hu

Design of Driver-Vehicle Interface to Reduce Mode Confusion for Adaptive Cruise Control Systems 67 
Adjunct Proceedings of the $7^{\text {th }}$ International Conference on Automotive User Interfaces and Interactive Vehicular Applications (AutomotiveUI '15), Sept. 1-3, 2015, Nottingham, United Kingdom

Sang Hun Lee and Hwisoo Eom

LED-A-pillars: Displaying Distance Information on the Cars Chassis

Alexander Meschtschjakov, Lukas Wanko and Fabio Batz

Adaptive Digital Sunshade: Blocking the Sun From Blinding the Driver

Alexander Meschtscherjakov, Hubert Scharfetter, Stefan Paul Kernjak, Nino Marcel Kratzer and Julian Stadon

Highly Automated Truck Driving - How Can Drivers Safely Perform Sport Exercises on the Go?

Natalie Richardson, Michael Sinning, Michael Fries, Sonja Stockert and Markus Lienkamp

Concept of a Reference Architecture for an Extendable In-vehicle Adaptive Recommendation Service

Nadine Walter, Tobias Altmüller and Klaus Bengler

On-wheel Finger Gesture Control for In-vehicle Systems on Central Consoles

Sang Hun Lee, Se-One Yoon and Jae Hoon Shin

Good Vibrations - Driving with a Haptic Pedal

Claudia Geitner, Stewart Birrell, Lee Skrypchuk, Claudia Krehl, Alex Mouzakitis and Paul Jennings

Field Studies to Investigate Safety Distance Violation with a Low-cost Observation System 106

Clemens Schartmüller and Andreas Riener

Deriving Future User Experiences in Autonomous Vehicle

Hyang Sook Kim, Sol Hee Yoon, Meen Jong Kim and Yong Gu Ji

eMotion: Retrospective In-Car User Experience Evaluation

Evangelos Niforatos, Evangelos Karapanos, Marc Langheinrich, Daniela Wurhofer, Alina Krischkowsky, Marianna Obrist and Manfred Tscheligi

LCTNav: A Method for Investigating Collaborative Navigation 124

Sandra Trösterer, Martin Wuchse, Axel Baumgartner, Bernhard Maurer, Magdalena Gärtner, Alexander Meschtscherjakov and Manfred Tscheligi

Evaluation of Historical Electric Vehicle (EV) Driving Data to Suggest Improvements in Driving Efficiency Benjamin Pichler and Andreas Riener

MaDSAV: Maintaining Driving Skills in Semi-Autonomous Vehicles

Alexander Meschtscherjakov, Rod McCall, Nicolas Louveton, Thomas Engel, Manfred Tscheligi and Vincent Koenig

An On-Road Study Involving Two Vehicles: Observed Differences between an Auditory and Haptic Lane Departure Warning System

Daniel Brown, Bryan Reimer, Bruce Mehler and Jonathan Dobres

Influence of In-Vehicle Eco-Driving Displays on Driver Behaviour

Peter Burns, Leanna Beluz, Marc Belzile, Vittoria Battista, Samuel Pedroso, James Knowles, Vijay Gill and Charles Crispim 
Adjunct Proceedings of the $7^{\text {th }}$ International Conference on Automotive User Interfaces and Interactive Vehicular Applications (AutomotiveUI '15), Sept. 1-3, 2015, Nottingham, United Kingdom

\section{Interactive Demos}

Simulator Telemetry (STING) and Head Up Display Designer Middleware for the NADS MiniSim

Driving Simulator .

Thomas Gable, Bruce Walker, Bhargav Rajendra and Fang He

DAZE: A Real-Time Situation Awareness Measurement Tool for Driving 158

Nikolas Martelaro, David Sirkin and Wendy Ju 
Adjunct Proceedings of the $7^{\text {th }}$ International Conference on Automotive User Interfaces and Interactive Vehicular Applications (AutomotiveUI '15), Sept. 1-3, 2015, Nottingham, United Kingdom

\section{Work in Progress}

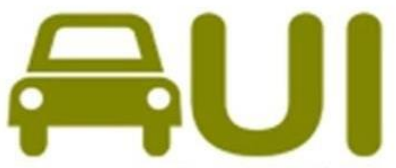

Automotive UI 2015

Nottingham, UK 
Adjunct Proceedings of the $7^{\text {th }}$ International Conference on Automotive User Interfaces and Interactive Vehicular Applications (AutomotiveUI '15), Sept. 1-3, 2015, Nottingham, United Kingdom

\section{Interactive Demo}

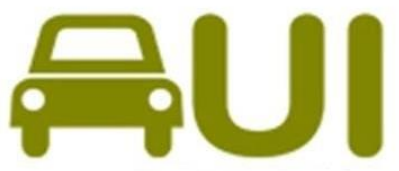

Automotive UI 2015

Nottingham, UK 